\title{
Variations saisonnières des performances de reproduction des brebis Ouled Djellal dans la région de Chlef, Algérie
}

\author{
Azdinia Zidane ${ }^{1 *}$ Mourad Taherti ${ }^{1}$ Leila Gadouche ${ }^{2}$ \\ Sarra Metlef ${ }^{3}$ Adda Ababou ${ }^{1}$
}

\section{Mots-clés}

Ovin, brebis Ouled Djellal, performance de reproduction, reproduction animale, printemps, automne, Algérie

(C) A. Zidane et al., 2021

\section{(c) (1)}

https://creativecommons.org/licenses/by/4.0/

Submitted: 27 November 2020

Accepted: 27 September 2021

Published: 20 December 2021

DOI: $10.19182 /$ remvt.36801

\section{Résumé}

La reproduction contribue directement à la rentabilité des élevages et les performances associées sont synthétisées dans des indicateurs économiques centraux dans l'analyse des performances globales des systèmes d'élevages. L'activité sexuelle et ainsi les performances reproductives des brebis sont affectées par des facteurs environnementaux, en premier lieu la photopériode. La brebis Ouled Djellal, naturellement davantage désaisonnée que d'autres races ovines, est peu sensible au facteur photopériodique, parvenant ainsi à maintenir des cycles sexuels pendant la majeure partie de l'année et exprimant peu d'anœstrus saisonnier. Les performances reproductives de cette race n'étant que partiellement exploitées, la présente étude a visé à les caractériser dans la région de Chlef, Algérie. Elle a concerné 834 brebis Ouled Djellal réparties dans 20 exploitations localisées dans différentes communes avec 417 brebis pour chaque saison. Les brebis étaient âgées de trois à cinq ans, et soumises à la photopériode et aux températures naturelles. Les paramètres reproductifs analysés à l'automne et au printemps ont été les taux de fertilité, de fécondité, de prolificité, de productivité numérique et de mortalités. A l'exception des mortalités qui ont montré une différence non significative entre I'automne et le printemps, les résultats sur les autres paramètres de performance ont mis en évidence une différence très significative $(p<0,0001)$ entre les deux saisons. Malgré ces différences entre saisons, dont les origines sont discutées, les performances reproductives analysées ont été globalement encourageantes pour améliorer la rentabilité des élevages de cette race.

- Comment citer cet article : Zidane A., Taherti M., Gadouche L., Metlef S., Ababou A., 2021. Seasonal variations in the reproductive performance of Ouled Djellal ewes in the Chlef region, Algeria. Rev. Elev. Med. Vet. Pays Trop., 74 (4): 193-198, doi: 10.19182/remvt.36801

\section{INTRODUCTION}

La reproduction et la survie des espèces sont liées à la coordination de divers mécanismes qui engendrent la production de gamètes, un comportement sexuel spécifique conduisant à l'accouplement et à la rencontre des gamètes, et in fine au développement d'embryons viables

\footnotetext{
1. Laboratoire bio-ressources naturelles, Département de biologie, Faculté des sciences de la nature et de la vie, Université Hassiba Benbouali de Chlef, BP151, Chlef 02000, Algérie.

2. Université des sciences et de la technologie Houari Boumédiène (USTHB), Faculté des sciences biologiques, Département de biologie et physiologie des organismes, El Alia, 16111 Bab Ezzouar Alger, Algérie.

3. Laboratoire bio-ressources naturelles, Département des sciences alimentaires et nutrition humaine, Faculté des sciences de la nature et de la vie, Université Hassiba Benbouali de Chlef, Chlef 02000, Algérie.

* Auteur pour la correspondance

Tél. : +213556887800 ; email : zidazdinia.2020@gmail.com
}

(Lamrani, 2008). Ces conditions sont également indispensables pour l'élevage car elles participent à sa rentabilité (Meyer, 2009). L'activité sexuelle ainsi que les performances reproductives des brebis sont affectées par des facteurs de leur environnement, principalement la photopériode. Chez certaines races plus désaisonnées, dont le lieu d'habitat est proche de l'équateur, la faible sensibilité au facteur photopériodique conduit les femelles à pouvoir maintenir des cycles sexuels pendant la majeure partie de l'année (Castonguay, 2018). C'est notamment le cas des brebis de race Ouled Djellal qui expriment peu ou pas d'anœstrus saisonnier.

De nombreux auteurs témoignent des bonnes qualités reproductives de la Ouled Djellal, de son bon comportement maternel et surtout de sa rusticité exceptionnelle à l'égard des conditions environnementales (Chellig, 1992). En Algérie, malgré son indéniable importance économique et sociale, l'élevage ovin est pénalisé par un faible niveau de technicité et une organisation des systèmes de production favorisant une conduite des activités de reproduction en pleine saison sèche, ceci 
visant à faire coïncider les mises bas avec le pic de végétation des premières pluies d'octobre (Arbouche et al., 2013). Toutefois, cette organisation empêche d'exploiter pleinement le potentiel des performances reproductives, notamment du fait d'une mauvaise préparation nutritionnelle des femelles autour de la période de lutte (Lamrani, 2008).

L'obtention de données fiables sur la conduite et la productivité des élevages utilisant cette race est encore aujourd'hui laborieuse. Ainsi, d'importantes investigations doivent être conduites afin de déterminer précisément les performances associées. Dans ce contexte, la présente étude a visé à caractériser les performances reproductives des brebis Ouled Djellal dans la région de Chlef, et à les comparer entre saisons d'automne (correspondant à la saison des pluies) et de printemps (correspondant à la saison sèche).

\section{MATERIEL ET METHODES}

\section{Site de l'étude et conduite des animaux}

La région de Chlef, organisée autour de la ville éponyme, est localisée dans le Tell occidental à 200 kilomètres à l'ouest d'Alger. Elle est caractérisée par un climat méditerranéen de type semi-aride avec une pluviométrie annuelle moyenne de 40,1 $\pm 5,7$ millimètres par mois.

L'expérimentation a concerné 834 brebis de race Ouled Djellal, réparties dans 20 exploitations localisées dans différentes communes, avec 417 brebis pour chaque saison étudiée (tableau I). Les résultats des performances reproductives des femelles mises à la lutte à l'automne (saison humide) sont représentés par le groupe «Printemps », et ceux des brebis mises à la lutte au printemps (saison sèche) par le groupe " Automne ». Les femelles retenues étaient des brebis âgées de trois à cinq ans, soumises à la photopériode et aux températures naturelles de la zone. Quelle qu'ait été la saison, les béliers étaient introduits dans les troupeaux au moment de la période de lutte pour une reproduction par la monte naturelle. Les élevages étaient conduits en mode semi-intensif avec une période de pâturage dans la journée et une période dans la bergerie en fin de journée où les brebis bénéficiaient d'une supplémentation à base de concentrés et d'un abreuvement $a d$ libitum. Durant les périodes où l'offre alimentaire au pâturage était jugée insuffisante, les brebis étaient gardées en bergerie et alimentées à base de foin naturel et de paille de blé.

Tableau I : Répartition des brebis Ouled Djellal dans les exploitations et selon la saison de lutte (Prin, groupe Printemps, $n=417$ ) ; Aut, groupe Automne, $\mathrm{n}=417$ ) en Algérie /// Distribution of Ouled Djellal ewes in the farms and according to the mating season (Prin: Spring group, $n=417)$; Aut: Fall group, $n=417$ ) in Algeria

\begin{tabular}{lll} 
Commune & Prin & Aut \\
\hline Chlef & 11 & 14 \\
Chettia & 13 & 15 \\
Ouled Fares & 18 & 17 \\
& 50 & 45 \\
Labiod Medjadja & 25 & 22 \\
Zeboudja & 22 & 18 \\
Bouzghaia & 16 & 13 \\
& 30 & 27 \\
& 21 & 25 \\
& 33 & 34
\end{tabular}

\begin{tabular}{lcc} 
Commune & Prin & Aut \\
\hline Tadjena & 40 & 38 \\
& 18 & 20 \\
& 30 & 26 \\
Sidi Akkacha & 14 & 15 \\
Abou El Hassan & 13 & 18 \\
Talassa & 10 & 15 \\
& 12 & 16 \\
& 15 & 14 \\
Sidi Abder-rahmane & 12 & 10 \\
Ténès & 14 & 15
\end{tabular}

\section{Paramètres enregistrés}

Un suivi spécifique a été mis en place afin de mesurer différents paramètres, créer une base de données et procéder aux analyses. L'objectif principal était d'obtenir des valeurs fiables pour les différents critères associés aux performances de reproduction. Ainsi le taux de fertilité apparente, soit l'indicateur de l'aptitude des femelles à concevoir au moins un agneau suite à la campagne de reproduction, a été calculé selon la formule : (nb. de femelles ayant mis bas) $\frac{(\text { nb. de femelles mises à la lutte) }}{2} \times 100$.

Le taux de fécondité, soit l'indicateur du nombre d'agneaux produits à l'échelle du troupeau suite à la campagne de reproduction, a été calculé selon la formule : (nb. de nouveau-nés) $\frac{\text { (nb. de femelles mises à la lutte) }}{} \times 100$.

Le taux de prolificité, soit l'indicateur de la taille moyenne d'une portée, a été calculé selon la formule : (nb. de nouveau-nés) $\frac{\text { (nb. de femelles ayant mis bas) }}{\text { (nb. de nouveau-nés) }} \times 100$.

Le taux de productivité numérique, soitl'indicateur du nombre d'agneaux viables produits par brebis suite à la campagne de reproduction, a été calculé selon la formule: (nb. de nouveau-nés élevés) $\frac{(\text { nb. de femelles mises à la lutte })}{100}$.

Le taux de mortalités, soit l'indicateur des pertes néonatales aussi bien chez les brebis que chez les agneaux, a été calculé selon la formule : (nb. de morts chez les nouveau-nés) (nb. de nouveau-nés)

Cette organisation a permis de s'adapter aux différents modes de conduite rencontrés, souvent associés à des niveaux de performances techniques très différents, mais également de dépasser le caractère trop fastidieux pour l'acquisition de certains enregistrements, notamment le contrôle des performances de reproduction. Elle a permis d'obtenir avec précision les valeurs réelles des indicateurs de performances recherchés. Les Ouled Djellal étant identifiées grâce à des boucles, des fiches individuelles ont également été établies pour ces femelles, avec des visites hebdomadaires pour chaque exploitation suivie.

\section{Analyses statistiques}

Le modèle statistique a consisté en un test paramétrique $t$ de Student visant à comparer les valeurs moyennes des différents paramètres entre saisons de mise bas au seuil de significativité fixé à $5 \%$. Ensuite une analyse en composantes principales (ACP) a été effectuée pour établir les relations entre les paramètres analysés et les saisons de mise bas. Les analyses ont été menées avec le logiciel XLSTAT version 2019.1 de Addinsoft. Toutes les données ont été présentées sous la forme de la moyenne plus ou moins l'écart-type.

\section{RESULTATS}

\section{Fertilité}

Les taux de fertilité ont été très significativement différents $(\mathrm{p}<0,0001)$ dans les deux groupes avec un taux moyen plus élevé dans le groupe Automne $(88 \pm 8 \%)$ que dans le groupe Printemps $(75 \pm 7 \%)$.

\section{Fécondité}

Les analyses ont mis en évidence une différence très significative $(\mathrm{p}<0,0001)$ du taux de fécondité selon la période de mises bas, avec une moyenne de $95 \pm 8 \%$ dans le groupe Automne contre $77 \pm 8 \%$ dans le groupe Printemps.

\section{Prolificité}

Les moyennes ont été différentes très significativement $(\mathrm{p}<0,0001)$ entre les deux groupes de mises bas avec $109 \pm 5 \%$ dans le groupe Automne contre $102 \pm 4 \%$ dans le groupe Printemps. 


\section{Productivité numérique}

Les analyses des taux de productivité numérique ont montré une différence très significative $(\mathrm{p}<0,0001)$ entre les groupes Automne $(93$ $\pm 8 \%)$ et Printemps $(74 \pm 8 \%)$.

\section{Mortalités}

Les moyennes des taux de mortalités ont montré une différence non significative $(p>0,05)$ entre les deux saisons de mise bas avec 3,0 $\pm 2,8 \%$ dans le groupe Automne et $1,9 \pm 2,1 \%$ dans le groupe Printemps. Cependant, alors que les valeurs des paramètres précédents étaient favorables au groupe Automne, les mortalités des agneaux ont été moins nombreuses dans le groupe Printemps.

L'ACP, prenant en considération la saison de mise bas en tant que variable supplémentaire (figure 1), a montré que les deux premiers axes expliquaient 93,41\% de l'information. Le premier axe avec 69,93\% de variabilité expliquée a montré une nette séparation entre les deux saisons de mise bas ; cet axe est corrélé positivement à la saison automnale et négativement à la saison printanière. L'analyse permet de souligner que les meilleures performances correspondaient à la lutte de printemps. Toutefois, le taux de mortalités n'a été lié à aucune des deux saisons de mise bas.

\section{DISCUSSION}

L'analyse des systèmes de production pratiqués par les éleveurs de la région de Chlef en fonction des rythmes et périodes de reproduction, associée au caractère désaisonné de la race Ouled Djellal, ainsi qu'à la répartition des agnelages, nous ont permis de caractériser deux saisons de mise bas distinctes : une mise bas de printemps correspondant aux performances associées à la lutte d'automne (groupe Printemps) et une mise bas d'automne correspondant aux performances associées à la lutte de printemps (groupe Automne).

Afin d'expliquer les variations des performances de reproduction entre les deux saisons, plusieurs facteurs peuvent être mis en avant, l'état corporel de l'animal étant probablement le plus important. Il

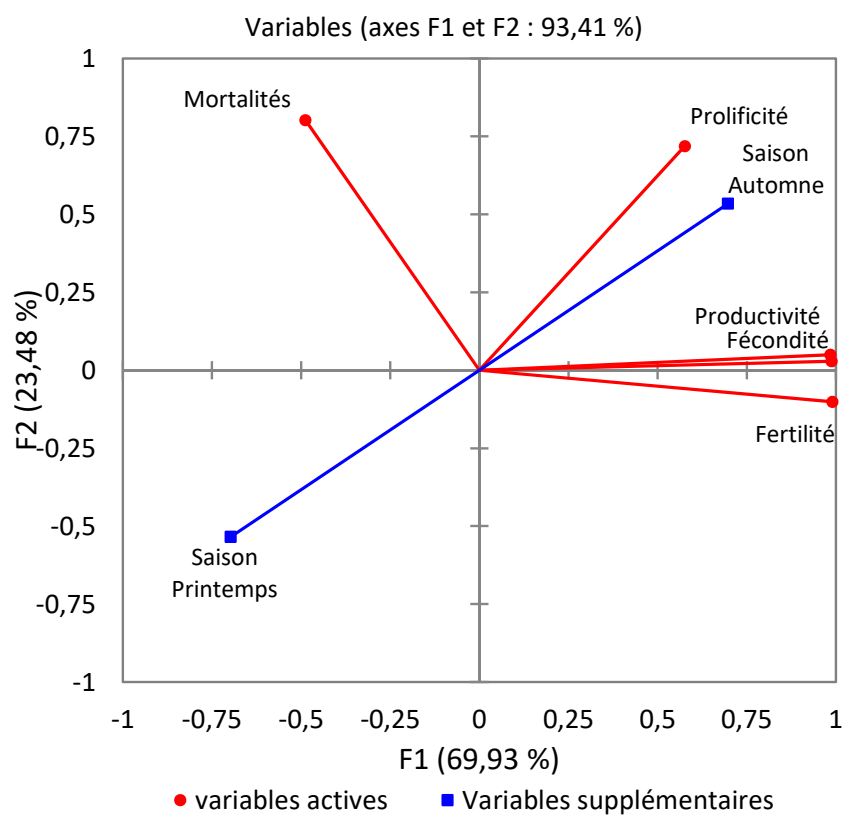

Figure 1 : Projection des variables sur les deux premiers axes de I'ACP chez la brebis Ouled Djellal selon la période de mise bas en Algérie /// Projection of variables on the first two axes of the PCA in Ouled Djellal ewes according to the period of lambing in Algeria semble ainsi nécessaire d'atteindre un état corporel satisfaisant au début de la lutte pour obtenir de bonnes performances, hypothèse privilégiée notamment par Taherti et Kaidi (2016) pour lesquels ce serait le déterminant principal du potentiel productif des brebis. Cependant, face à la réticence de certains éleveurs vis-à-vis de l'enregistrement de cette variable, aucune notation de l'état corporel des femelles n'a été réalisée durant l'expérimentation.

Au sein des exploitations enquêtées, l'état corporel des animaux au moment de la lutte n'était pourtant pas le facteur déterminant dans l'organisation des pratiques des éleveurs qui privilégiaient une mise à la reproduction au printemps, en saison sèche, pour des agnelages majoritairement en automne, en saison humide (Zidane et Ababou, 2017 ; Hadef, 2018). Pour ces éleveurs, le facteur prépondérant était le coût des saillies moindre au printemps qu'en automne. Si cette organisation favorise un environnement nutritionnel propice à la période des mises bas, elle contribue par ailleurs aussi à l'expression des impacts négatifs d'une sous-nutrition sur les évènements reproductifs associés à la période de la lutte. En conséquence, les brebis sont maintenues la plupart du temps en bergerie et les éleveurs cherchent à faire coïncider le plus longtemps possible leurs stocks de fourrages disponibles et les besoins des animaux. Ceux-ci bénéficient alors de fourrages verts et d'une complémentation, ainsi que de périodes de pacage sur chaumes de céréales en été. Cette organisation du rationnement alimentaire, principalement à base de paille et de foin, ne permet pas d'éviter des périodes de restrictions. Si elle ne favorise pas de gain de poids significatif, elle semble néanmoins agir comme le flushing et permettre aux brebis d'exprimer de meilleures performances lors de l'agnelage d'automne. Quant aux brebis mises à la reproduction à l'automne, elles bénéficient d'une période de pâturage plus longue qui correspond aussi à une période d'épuisement des pâturages et semble ainsi avoir un effet moins favorable sur les performances lors de l'agnelage de printemps.

\section{Fertilité}

Il est admis que les ovins sont capables de présenter, en monte naturelle, un taux de fertilité de $92 \%$. D'autre part, certains auteurs indiquent dans des conditions d'études comparables qu'une fertilité moyenne de $70 \%$ à $80 \%$ après saillie naturelle peut être considérée comme normale à bonne en automne et comme bonne à très bonne au printemps (Provost et al., 1980). Nos résultats pour les mises bas à l'automne ont été similaires à ceux rapportés pour les brebis Béni Guil au Maroc (Bourfia et Touchberry, 1993), Noires de Thibar en Tunisie (Zaiem et al., 2000), et Ouled Djellal dans les hauts plateaux d'Algérie (Dekhili, 2014). D'autre part, les résultats de fertilité observés pour les mises bas de printemps s'accordaient avec ceux de Benyounes et al. (2013a) et de Belhadia et al. (2020) obtenus chez des brebis Taâdmit en Algérie. Toutefois, pour cette même période et aussi en Algérie, les performances de fertilité observées dans notre étude ont été meilleures que celles de $83 \%$ chez la même race dans la région de Biskra (Mefti Korteby et al., 2017) et de 44,8 \% chez la Rembi dans la région de Tiaret (Khiati, 2013). Selon Chafri et al. (2008) ces meilleurs résultats associés à la saison d'automne sont à imputer à une préparation alimentaire adéquate (assimilable à un flushing) au cours des semaines précédant la lutte, facteur favorable à une bonne fertilité. De plus, Atti et Abdennebi (1995) montrent que l'état corporel des femelles est un facteur déterminant dans l'obtention de bonnes performances de reproduction en analysant la fertilité des brebis en fonction de leur note d'état corporel au moment de la lutte.

\section{Fécondité}

Les résultats obtenus pour la fécondité à l'issue de la lutte de printemps ont concordé avec ceux rapportés aussi chez des brebis 
Ouled Djellal en Algérie par Dekhili (2010) dans la région de Sétif, Arbouche et al. (2013) dans la région de Bordj Bou Arreridj, et Abaidia et al. (2020) dans la région de Tébessa. Ils ont été toutefois très différents du taux de $106 \%$ relevé chez les brebis laitières Sicilo-Sardes en Tunisie (Selmi et al., 2009), et de $108 \%$ obtenu chez les brebis Ouled Djellal de la région de Souk-Ahras en Algérie (Benyounes et al., 2013a). Ces différences peuvent provenir du mois et de la saison de saillie qui ont un effet très marqué sur cette performance (Dekhili, 2010), de la gestion de l'alimentation, et de l'adaptation spécifique de la brebis Ouled-Djella et des systèmes d'élevages associés. Quant au taux de fécondité pour les brebis issues d'une lutte automnale, il a été le même que ceux rapportés chez la brebis Taâdmit dans la région de Souk-Ahras (Benyounes et al., 2013a) et chez la Noire de Thibar en Tunisie (Ben Salem et al., 2009).

\section{Prolificité}

La prolificité est considérée comme un paramètre zootechnique primordial pour la rentabilité en élevage ovin. Nos résultats relatifs à la prolificité des brebis issues de la lutte de printemps ont été semblables à ceux de Chellig (1992) chez des brebis Ouled Djellal et de Khiati (2013) chez des brebis Rembi à Tiaret en Algérie, ainsi que ceux de Buldgen et al. (1992) chez des brebis améliorées dans la région du Sine-Saloum au Sénégal. Toutefois, El Fadili (2005) rapporte une valeur de $220 \%$ chez des brebis D'Man, caractérisées par une prolificité exceptionnelle, dans la zone Bour Atlantique du Maroc (Boujenane, 2003). A l'inverse, la Ouled Djellal n'étant pas connue pour être une brebis prolifique (Lamrani et al., 2008), cette différence s'expliquerait surtout par un facteur génétique. Concernant le taux de prolificité associé à la lutte automnale, il a été en accord avec ceux rapportés chez les brebis Toronké au Mali (Kouriba et al., 2004) et Taâdmit en Algérie (Benyounes et al., 2013a ; Belhadia et al., 2020).

Comme le montrent Scaramuzzi et al. (2006), les résultats peuvent expliquer la relation directe entre la prolificité d'un troupeau et son état général avant la lutte. Ainsi, l'alimentation des animaux ayant été principalement basée sur des parcours pauvres durant la période de lutte en automne, ce paramètre s'est répercuté négativement sur la prolificité des brebis ayant mis bas au printemps, comme observé par Chikhi et Boujenane (2003).

\section{Productivité numérique}

Etant l'outil d'appréciation du niveau de performances d'un troupeau, la moyenne de productivité numérique de $93 \%$ associée à la lutte printanière observée dans notre étude a été la même que celle des brebis de la même race dans la région de Sétif (Dekhili et Benkhlif, 2005) et des brebis Sicilo-Sardes en Tunisie (Selmi et al., 2009). Cependant, elle a dépassé largement celle de $73 \%$ chez des brebis Ouled Djellal dans la même zone d'étude (Taherti et Kaidi, 2018). Les résultats moyens des brebis mises à la lutte à l'automne ont été supérieurs à ceux de 68,9\% chez des brebis Ouled Djellal croisées avec des béliers D'man (Adaouri et al., 2017). En termes de productivité numérique, Niaré (1995) souligne l'effet de la saison d'agnelage qui se manifeste par la survie des jeunes dans la portée. D'autre part, la brebis Ouled Djellal présente un comportement reproductif différent lorsqu'elle est placée dans des systèmes d'élevage différents (Taherti et Kaidi, 2018). En résumé, l'année, la saison et le mois de lutte ont une incidence sur le taux de productivité (Dekhili et Aggoun, 2006). Ainsi, les différences observées dans notre étude étaient probablement la conséquence des modes de conduite de la reproduction et de l'alimentation.

\section{Mortalités}

Quelle qu'ait été la saison, nous avons relevé des taux de mortalités des agneaux de 0-7 jours similaires à ceux de Boubekeur et al.
(2019) chez des brebis D'man en Algérie. Ces taux ont été très faibles comparés à celui de 14,6\% chez des brebis laitières Sicilo-Sardes en Tunisie (Selmi et al., 2009), et de 21,4\% chez des brebis Ouled Djellal en Algérie (Chemmam et al., 2014). Ainsi, contrairement à l'ensemble des autres performances, les mortalités des agneaux ont diminué au printemps par rapport à l'automne. Stafford et al. (2007) rapportent que la survie des agneaux après la naissance dépend de nombreux facteurs. Dans notre étude, le taux plus important des mortalités des agneaux nés à l'automne a été surtout dû au froid, corroborant ainsi les constatations de Prud'hon (1971) selon lequel les mortalités maximales en hiver sont imputables au froid qui peut perturber le réflexe des tétées et l'instinct maternel des brebis. Elles peuvent aussi être la conséquence d'une mauvaise prise en charge des agneaux durant la première semaine suivant la naissance (Benyounes et al., 2013b). Généralement, les pertes les plus importantes sont observées durant les périodes de fortes pluies (Boucherit, 1985). Cependant, les conditions climatiques clémentes du printemps, associées à certains soins apportés par les éleveurs aux nouveau-nés ont permis leur survie et ont ainsi minimisé le taux de mortalités durant cette saison.

\section{CONCLUSION}

Les variations des performances reproductives relevées au cours des saisons provenaient probablement d'une interaction temporelle complexe entre les disponibilités alimentaires de la région, l'organisation du rationnement et le degré de mobilisation des réserves corporelles selon le stade physiologique. Les faibles performances reproductives des brebis Ouled Djellal mises à la lutte à l'automne ont été probablement la conséquence d'une sous-alimentation durant cette saison. Le contrôle de la saison de lutte s'avère ainsi primordial pour mettre à profit les ressources alimentaires qui sont très saisonnées dans la région. Les différences de rendements constatées étaient la répercussion des différents comportements développés par la brebis Ouled Djellal par rapport aux modes de conduite des activités de reproduction mais surtout de l'alimentation qui a eu un effet majeur sur les performances saisonnières de reproduction. Bien que des différences au niveau du bilan reproductif des brebis Ouled Djellal dans la région de Chlef aient été évidentes, le rendement reproductif à travers ses composantes (fertilité, fécondité prolificité, productivité numérique et mortalités) a été globalement encourageant pour cette race, comparé à d'autres races en Algérie ou dans d'autres pays. Il ressort aussi l'intérêt de mettre la Ouled Djellal à la reproduction durant toute l'année ; sa capacité de conception continue est un atout permettant d'avoir des mises bas tout au long de l'année, ou de les moduler en fonction des besoins (automne-printemps) et des disponibilités fourragères. La Ouled Djellal présente de nombreux avantages dont une forte capacité productive des troupeaux dans une perspective d'amélioration de la production de viande rouge en Algérie.

\section{Remerciements}

Les auteurs remercient sincèrement tous les éleveurs qui ont contribué à la réalisation de cette étude.

\section{Déclaration de conflits d'intérêts}

Les auteurs déclarent que l'étude a été réalisée sans conflit d'intérêts.

\section{Déclaration des contributions des auteurs}

AZ, MT et SM ont conçu et planifié l'étude ; AZ et MT ont collecté les données ; $\mathrm{AZ}$ a rédigé la première version du manuscrit ; $\mathrm{AA}$ a analysé et interprété les données; LG a fait la révision critique du manuscrit. Tous les auteurs ont lu et approuvé le manuscrit dans sa forme actuelle. 


\section{REFERENCES}

Abaidia A., Mebirouk-Boudechiche L., Chaker-Houd K., 2020. Effet d'une addition de rebuts de dattes sur les performances de brebis Ouled Djellal et leurs agneaux en milieu steppique. Livest. Res. Rural Dev., 32 (38)

Adaouri M., Mefti Korteby H., Triki S., Lebied M., Djouadi S., Balouli N., Sebbag L., 2017. Effets d'un croisement D'man x Ouled Djellal sur la reproduction des brebis et la croissance des $\mathrm{F}_{1}$ (première lutte). Livest. Res. Rural Dev., 29: 206

Arbouche R., Arbouche H.S., Arbouche F., Arbouche Y., 2013. Facteurs influençant les paramètres de reproduction des brebis Ouled Djellal. Arch. Zootecn., 62 (238): 311-314, doi: 10.4321/S0004-05922013000200020

Atti N., Abdennebi L., 1995. Etat corporel et performances de la race ovine Barbarine. CIHEAM, Cahiers Opt. Méditerr., 6: 75-80

Belhadia M.A., Fantazi K., Benaouina H., Kada M., Belhandouze T., Housseini N., 2020. Performances de reproduction de brebis Taâdmit avec effet mâle et synchronisation des chaleurs. Livest. Res. Rural Dev., 32: 123

Ben Salem I., Rekik M., Hammami H., Ben Hamouda M., Aloulou R., Saâdoun L., 2009. Non-genetic factors of variation of the productivity of the Noire de Thibar ewe. Rev. Elev. Med. Vet. Pays Trop., 62 (1): 59-66, doi: 10.19182/remvt.10095

Benyounes A., Rezaiguia M., Lamrani F., 2013a. Rendement reproductif des brebis Ouled Djellal et Taadmit élevées dans la région Nord-Est d'Algérie. Livest. Res. Rural Dev., 25: 199

Benyounes A., Rezaiguia M., Lamrani F., 2013b. Effet de la saison d'agnelage sur la mortalité des agneaux chez les races ovines Ouled Djellal et Taâdmit élevées dans le Nord-est d'Algérie. Rev. Agric., 5: 5-9

Boubekeur A., Benyoucef M.T., Benidir M., Slimani A., Maaref A., Lounassi M., 2019. Qualités reproductives des brebis D'Man en oasis algériennes. Livest. Res. Rural Dev., 3: 129

Boucherit N., 1985. Contribution à l'étude de la mortalité périnatale chez les agneaux : influence des facteurs zootechniques et causes de la mortalité. Thèse Doct., IAV Hassan II, Rabat, Maroc, 132 p.

Boujenane I., 2003. Amélioration génétique ovine au Maroc : contraintes et voies d'amélioration. Terre et vie, 70: 1-4

Bourfia M., Touchberry R.W., 1993. Diallel cross of three Moroccan breeds of sheep. I. lamb growth and carcass traits. J. Anim. Sci., 71 (4): 870-881, doi: 10.2527/1993.714870x

Buldgen A., Dieng A., Ducrot D., Dumont D., Compère R., 1992. Productivité des élevages villageois de moutons du bassin arachidier sénégalais. FAO, Rome, Italie, 11 p (Série 72)

Castonguay F., 2018. La reproduction chez les ovins. Université Laval, Edition Mars, Québec, Canada, 145 p.

Chafri H., Mahouachi M., Ben Hamouda M., 2008. Effet du niveau alimentaire après mise bas sur le développement de la fonction reproductive chez I'agneau de race prolifique D'man : Développement testiculaire et déclenchement de la puberté. Rencontres Rech. Rumin., 15: 394

Chellig R., 1992. Les races ovines algériennes. OPU, Alger, Algérie, 80 p.

Chemmam M., Meftah N., Boudechiche M.L., 2014. Effets de l'avancement de la saison sexuelle sur les performances de reproduction et le poids des agneaux Ouled Djellal au sevrage dans le nord-est de l'Algérie. Livest. Res. Rural Dev., 26: 142

Chikhi A., Boujenane I., 2003. Genetic and phenotypic parameters of reproductive performance of Boujaâd and Sardi ewes. Rev. Elev. Med. Vet. Pays Trop., 56 (1-2): 83-88, doi: 10.19182/remvt.9880

Dekhili M., Benkhlif R., 2005. Bilan portant sur les performances reproductives d'un troupeau de brebis Ouled Djellal. Rencontres Rech. Rumin., 12: 162

Dekhili M., Aggoun A., 2006. Paramètres génétiques de la productivité numérique des brebis Ouled-Djellal. Rencontres Rech. Rumin., 13: 221
Dekhili M., 2010. Fertilité des élevages ovins type « Hodna » menés en extensif dans la région de Sétif. Rev. Agron., 1 (0): 1-7

Dekhili M., 2014. Paramètres phénotypiques et génétiques de la reproduction de la brebis Ouled-Djellal (Algérie). Arch. Zootech., 63 (242): 269-275, doi: 10.4321/S0004-05922014000200005

El Fadili M., 2005. La race prolifique ovine D'man : productivité et voies de valorisation en dehors de l'oasis. Transfert Techn. Agri., 130: 1-4

Hadef A., 2018. Caractéristiques de l'activité sexuelle chez les brebis pâturant les prairies littorales de l'extrême nord est algérien. Rev. Algérienne Sci. Sect. A (1) : 27-31

Khiati B., 2013. Etude des performances reproductives de la brebis de race Rembi. Thèse Doct., Université d'Oran, Algérie, 188 p.

Kouriba A., Nantoumé H., Ogola D., 2004. Caractères de reproduction et mortalité des jeunes moutons Toronké à la station de recherches zootechniques de Kayes. Tropicultura, 22 (3): 134-138

Lamrani F., 2008. Etude de la cyclicité des agnelles et des possibilités de maîtrise de la reproduction des femelles Ouled Djellal combinées à l'effet de l'emploi répété de la PMSG sur leurs aptitudes reproductives. Thèse Doct. Université Badji Mokhtar, Annaba, Algérie, 179 p.

Lamrani F., Benyounes A., El Bouyahiaoui R., Toumi Feddaoui K., Sebbagh L. 2008. Effet du mode d'induction et de synchronisation des chaleurs sur le rendement reproductif des brebis Ouled Djellal. Rech. Agronom., 12 (21) 59-71

Mefti Korteby H., Koudri Z., Saadi M.A., 2017. Caractérisation des performances de la race ovine algérienne Ouled Djellal type Djellalia dans des conditions steppiques. Nat. Technol., 9 (2) : 1-5

Meyer C., 2009. Les variations saisonnières de la reproduction des bovins domestiques en zone tropicale, synthèse. CIRAD, Montpellier, France 26 p.

Niaré T., 1995. Pre-weaning growth of lambs and productivity in SudanoSahelian traditional area in Mali. Rev. Elev. Med. Vet. Pays Trop., 48 (2): 195-202, doi: 10.19182/remvt.9472

Provost A., Charray J., Coulomb J., Haumesser J.B., Planchenault D., Pugliese P.L., 1980. Les petits ruminants d'Afrique Centrale et d'Afrique de l'Ouest synthèse des connaissances actuelles. GERDAT-IEMVT, ministère de la Coopération, Maisons-Alfort, France, 295 p.

Prud'hon M.H., 1971. Etude de paramètres influençant la fécondité des brebis et la mortalité des agneaux d'un troupeau de race Mérinos d'Arles. Thèse Sciences appliquées, Université Sciences et techniques de Languedoc, Montpellier, France, 157 p.

Scaramuzzi R.J., Campbell B., Downing J.A., Kendall N.R., Khaldi M., MunozGutiérrez M., Somchit A., 2006. A review of the effects of supplementary nutrition in the ewe on the concentrations of reproductive and metabolic hormones and the mechanisms that regulate folliculogenesis and ovulation rate. Reprod. Nutr. Dev. 46 (4): 339-354. doi: 10.1051/rnd:2006016

Selmi H., N'cir M., Rekik B., Ben Gara A., Rouissi H., 2009. Performances de reproduction et de production en relation avec l'état sanitaire des brebis laitières Sicilo-Sarde. Livest. Res. Rural Dev., 21: 130

Stafford K.J., Kenyon P.R., Morris S.T., West D.M., 2007. The physical state and metabolic status of lambs of different birth rank soon after birth. Livest. Sci., 111 (1-2): 10-15, doi: 10.1016/j.livsci.2006.10.018

Taherti M., Kaidi R., 2016. Body condition variations and sheep breeding systems in the region of Chlef, Algeria. Rev. Elev. Med. Vet. Pays Trop., 69 (3): 105-109, doi: 10.19182/remvt.31193

Taherti M., Kaidi R., 2018. Productivité de la brebis Ouled Djellal selon le mode de conduite de la reproduction. J. Sci. Lib., 19 (1): 47-58, doi: 10.22453/LSJ-019.1.047-058

Zaiem I., Chemli J., Slama H., Tainturier D., 2000. Amélioration des performances de reproduction par l'utilisation de la mélatonine chez la brebis à contre-saison en Tunisie. Rev. Méd. Vét., 151 (6): 517- 522

Zidane A., Ababou A., 2017. Variations hormonales saisonnières de brebis Ouled Djellal dans la région de Chlef, Algérie. Livest. Res. Rural Dev., 29: 239 


\section{Summary}

Zidane A., Taherti M., Gadouche L., Metlef S., Ababou A. Seasonal variations in the reproductive performance of Ouled Djellal ewes in the Chlef region, Algeria

Reproduction contributes directly to the profitability of the farms and the associated performances are synthesized in central economic indicators in the analysis of the global performances of the breeding systems. The sexual activity and thus the reproductive performance of ewes are affected by environmental factors, primarily the photoperiod. The Ouled Djellal ewe, naturally more often bred out of season than other sheep breeds, is not very sensitive to the photoperiod factor, thus managing to maintain sexual cycles during most of the year and expressing little seasonal anestrus. As the reproductive performance of this breed is only partially exploited, the present study aimed to characterize it in the region of Chlef, Algeria. It involved 834 Ouled Djellal ewes distributed in 20 farms located in different municipalities with 417 ewes for each season. The ewes were three to five years old, and subjected to photoperiod and natural temperatures. The reproductive parameters analyzed in the fall and spring were fertility, fecundity, conception rate, numerical productivity and mortality. With the exception of mortality, which showed a non-significant difference between fall and spring, the results on the other performance parameters showed highly significant differences $(p<0.0001)$ between the two seasons. In spite of these differences between seasons, whose origins are discussed, the reproductive performances analyzed were globally encouraging to improve the profitability of this breed.

Keywords: sheep, Ouled Djellal ewes, reproductive performance, animal reproduction, spring, autumn, Algeria

\section{Resumen}

Zidane A., Taherti M., Gadouche L., Metlef S., Ababou A. Variaciones estacionales de los rendimientos de reproducción de las ovejas Ouled Djellal en la región de Chlef, Argelia

La reproducción contribuye directamente a la rentabilidad de los criaderos y los rendimientos asociados son sintetizados mediante los indicadores económicos centrales en el análisis de los rendimientos globales de los sistemas de cría. La actividad sexual y por ende los rendimientos reproductivos de las ovejas se ven afectados por factores ambientales, en primer lugar, el fotoperiodo. La oveja Ouled Djellal, naturalmente menos dependiente de la estacionalidad que otras razas ovinas, es poco sensible al factor fotoperiódico, logrando así mantener ciclos sexuales durante la mayor parte del año y expresando pocos anestros estacionales. Siendo los rendimientos reproductivos de esta raza sólo parcialmente explotados, el presente estudio pretendió caracterizarlos en la región de Chlef, Argelia. Este concernió 834 ovejas Ouled Djellal repartidas en 20 explotaciones localizadas en diferentes comunidades con 417 ovejas para cada estación. Las ovejas tenían de tres a cinco años de edad y estaban sometidas al fotoperiodo y a las temperaturas naturales. Los parámetros reproductivos analizados durante el otoño y la primavera fueron las tasas de fertilidad, de fecundidad, de prolificidad, de productividad numérica y de mortalidad. Con excepción de la mortalidad que mostró una diferencia no significativa entre el otoño y la primavera, los resultados sobre los otros parámetros de rendimiento evidenciaron una diferencia muy significativa $(p<0,0001)$ entre las dos estaciones. A pesar de estas diferencias entre estaciones, cuyos orígenes son discutibles, los rendimientos reproductivos analizados fueron globalmente alentadores para el mejoramiento de la rentabilidad de los criaderos de esta raza.

Palabras clave: ovino, oveja Ouled Djellal, reproductividad, reproducción animal, primavera, otoño, Argelia 